\section{Efeitos de bebidas alcóolicas em mães lactantes e suas repercussões na prole}

\section{The effect of alcoholic beverages in nursing mothers and their impact on children}

Maria Goretti Pessôa de Araújo Burgos 1

Maria do Carmo Medeiros 2

Francisca Martins Bion 3

Débora Catarine Nepomuceno de Pontes Pessoa 4

1 Laboratório de Nutrição Experimental. Departamento de Nutrição. Centro de Ciências da Saúde da Universidade Federal de Pernambuco. Rua Prof. Nelson Chaves, s. n. Cidade Universitária. Recife, Pernambuco, Brasil. CEP 50.970-901

2-4 Departamento de Nutrição. Centro de Ciências da Saúde da Universidade Federal de Pernambuco

\begin{abstract}
A study review on the effects of alcoholic beverage consumption in suckling children and analysis of multiple metabolic, nutritional and psychological conditions in mothers and newborns were accomplished. These emphasized the need of clinical and nutritional guidance in prenatal and childcare clinics related to the risk of alcoholic beverages intake, in any quantity by pregnant women and nursing mothers.

Key words Lactation, Alcoholism, Alcoholic beverages, Breast feeding, Nutritional status.
\end{abstract}

Resumo Foi feita uma revisão de estudos sobre os efeitos ocasionados pelo consumo de bebidas alcóolicas por lactantes, analisando os múltiplos distúrbios metabólicos, nutricionais e psicológicos evidenciados no organismo materno e dos recém-nascidos. É enfatizada a necessidade de orientações clínico-nutricionais nos serviços de pré-natal e puericultura acerca dos riscos da ingestão de bebidas alcoólicas em qualquer quantidade, por mães no período de gestação e aleitamento.

Palavras-chave Lactação, Alcoolismo, Bebidas alcóolicas, Aleitamento materno, Gravidez, Estado nutricional 


\section{Introdução}

As bebidas alcóolicas constituem uma importante fonte de calorias na dieta de adultos e adolescentes nos países desenvolvidos e naqueles em desenvolvimento.1,2 Aproximadamente 4-6\% das calorias ingeridas pela população mundial são provenientes do etanol contido nas bebidas alcoólicas, segundo dados resultantes de inquéritos de consumo alimentar. Nos dependentes de álcool e nos grandes bebedores, mais de 50\% das calorias consumidas diariamente é proveniente do etanol, substância alimentar com valor energético de $29,7 \mathrm{kj} / \mathrm{g}(7,1 \mathrm{kcal} / \mathrm{g}){ }^{3}$ O etanol é a droga de que mais se abusa, no Brasil e no mundo.4,2 Ingerido em excesso, provoca lesões peculiares no fígado e em outros órgãos, sendo responsável por alto índice de mortalidade nos países desenvolvidos. 5

\section{Fatores nutricionais e consumo de álcool}

São bastante controvertidos os achados a respeito dos efeitos benéficos do consumo de bebidas alcoólicas para a saúde, como por exemplo redução do colesterol das lipoproteínas de alta densidade, (C-HDL) provenientes da participação do etanol na dieta, quando utilizado com moderação (1-2 doses/dia). ${ }^{4}$ Por outro lado, são observadas interações entre o seu consumo excessivo com a biodisponibilidade e metabolismo de nutrientes, conforme mostrado por vários autores. $4,6-11$

Quanto à quantidade de calorias consumidas, ressalta-se que uma dose de bebida alcóolica contém entre 70 e 100kcal de etanol e outros carboidratos; consequentemente oito a dez doses podem proporcionar mais de $1.000 \mathrm{kcal} / \mathrm{dia}$, embora estas não sejam eficientemente utilizadas como combustível e não sejam armazenadas sob forma de gordura. ${ }^{8}$ Assim o etanol não é uma boa fonte de nutrientes, razão porque as calorias provenientes do seu consumo são denominadas "calorias vazias".

Sabe-se que, nos dependentes de álcool, a absorção das vitaminas pelo intestino delgado, por transporte ativo ou por alteração do armazenamento hepático, pode ser deficiente; isto ocorre com o ácido fólico, vitaminas B6, e B1, niacina e vitaminas A e D.12 Pode também ocorrer baixo nível sangüíneo de potássio, magnésio, cálcio, zinco, fósforo e alterações no magnésio intracelular devido a uma deficiência alimentar e/ou distúrbios do equilíbrio ácidobásico durante a ingestão alcoólica excessiva, na abstinência do álcool.12

Uma sobrecarga de etanol no indivíduo em jejum tende a produzir hipoglicemia transitória no espaço de seis a 36h, secundariamente às ações agudas do etanol sobre a gliconeogênese. Esse distúrbio é exacerbado por uma dieta deficiente e por patologias hepáticas e pancreáticas. 13

As interações entre o álcool e o estado nutricional são complexas, com os estudos indicando que o etanol é uma fonte eficaz de calorias, mas o seu consumo excessivo produzindo diminuição do peso corporal. Neste contexto, a perda de peso ocorre provavelmente devido ao aumento na degradação das proteínas musculares com balanço negativo de nitrogênio e água. ${ }^{3} \mathrm{O}$ consumo de álcool altera o metabolismo intermediário dos carboidratos e lipídeos, 13 e o metabolismo das vitaminas, 3 (por modificação da sua absorção) e em alguns casos, o seu armazenamento (aumentando sua excreção).14

\section{Associação entre gestação e bebidas alcóolicas}

Historicamente o álcool tem sido relacionado aos efeitos adversos sobre os recém-nascidos. Citações bíblicas já referiam proibições ao consumo de cerveja e de vinho por gestantes, sendo ainda norma proibitiva em algumas cidades da antigüidade o brinde nupcial com bebidas alcóolicas, no sentido de prevenir efeitos teratogênicos já na concepção. $15 \mathrm{Na}$ Grécia antiga os filósofos discutiam os possíveis efeitos deletérios do álcool sobre os filhos de mães que consumiam bebidas alcóolicas durante a gravidez. ${ }^{16}$

Estudos clínicos e experimentais 17-19 têm relatado que o consumo de bebidas alcóolicas durante a gestação causa malformação e retardo do crescimento, caracterizando 20 a síndrome fetal pelo álcool, cujas características mais importantes são: retardo do crescimento pré e pós-natais; alterações do sistema nervoso central, como microencefalia; alterações faciais, como hipoplasia facial e microftalmia. São ainda desconhecidos alguns dos principais mecanismos pelos quais o álcool induz tais efeitos, e vários autores 21,22 sugeriram que estes efeitos ocorrem através de mecanismos metabólicos atuando nos distintos estágios pré e pós-natais. Possivelmente estariam envolvidos no processo fatores maternos e nutricionais, entre outros.

\section{Efeitos do álcool na lactação}

Os efeitos decorrentes do uso de substâncias como etanol, cafeína, nicotina e outras, permitidos socialmente, vêm sendo investigados por vários autores, 
com evidências de que o álcool passa ao leite materno (LM) em grandes proporções, alterando a produção, o volume, o aroma, a composição e a excreção láctea, provocando efeitos deletérios no recém-nascido $(\mathrm{RN}) .23-30$

A maioria das gestantes tem conhecimento de que não deve consumir álcool neste período, entretanto o mesmo não ocorre com relação ao período de lactação, uma vez que a cerveja tradicionalmente e, em menor grau, o vinho, são recomendados como lactogôgos, fontes de vitaminas do complexo B e capazes de provocar sensação de repouso no binomio mãe/filho. ${ }^{31}$ Menella e Beauchamp32 investigaram os efeitos do álcool no lactente, evidenciando que o odor do LM é modificado imediatamente após a ingestão alcoólica. Assim, o lactente, apesar de sugar de forma mais vigorosa, ingere menos leite por tempo de sucção, fato não percebido pelas mães.

Segundo alguns autores, ${ }^{33-36}$ o sistema imunológico é afetado pela ingestão alcoólica na lactação, sendo observado déficit, a longo prazo, na imunidade celular e no sistema nervoso, indicando sensibilidade ao álcool em etapas precoces do desenvolvimento. 31

Alguns efeitos são evidenciados nas mães em decorrência da ingestão de álcool na gestação-lactação. As variações no peso feminino também afetam a desintoxicação alcoólica, as mulheres magras necessitando de maior tempo para metabolizar a mesma quantidade de álcool. ${ }^{31}$

Por outro lado observa-se que a absorção do etanol (após grupamento por idade, peso e etnia), é menor em curto período de tempo do que em não nutrizes. 37 Ademais a alcoolemia, que é semelhante ao teor de álcool no LM, é menor nas lactantes, e, consequentemente, a taxa de captação de álcool pelo fígado deve ser diferente, devido à menor quantidade de álcool circulante.

As pesquisas pioneiras de Lawton 38 evidenciaram que o teor de álcool no LM é diretamente proporcional ao do sangue, e esse teor não é afetado pela sucção ou aleitamento pré ou pós-ingestão de álcool. Assim à medida que a droga penetra no sangue, cresce a sua concentração.

Schuckit 12 mostrou uma relação entre o tempo de metabolização de bebidas alcoólicas a partir do peso de mulher não lactante. Esta relação indica que, enquanto uma mulher normal de $45 \mathrm{~kg}$ ingerindo 200ml de bebida despende 3,1 horas para eliminação corporal, outra de $72 \mathrm{~kg}$ despenderia 1,9 horas.

Por outro lado, nas lactantes observam-se redução no consumo alimentar e no peso corporal,21,39 e acúmulo de lipídeos nas mamas. 40 Pesquisa realizada, em 1989, no Instituto Nacional de Saúde Infantil e Desenvolvimento Humano nos Estados
Unidos, detectou diferenças significativas no desenvolvimento motor de $\mathrm{RN}$ de mães que bebiam de forma regular. 30 Efeitos tóxicos do uso de álcool levam a alterações, que dependem da idade da criança (o fígado imaturo será o mais afetado), do Índice de Massa Corporal (IMC) materno, da quantidade e concentração da bebida consumida, do período de jejum ou pós-prandial e da quantidade de gordura consumida. 41

Redução pondo-estatural e aumento da mortalidade neonatal, foram encontrados por diversos autores.22,39,41-46 Várias alterações metabólicas e hormonais maternas e consequentemente na composição do LM, bem como da metabolização do etanol, repercutem de forma significativa no desenvolvimento da prole. 43 Foram também observadas alterações nos RNs após consumo de $20 \%$ de álcool, pelas mães, com redução do peso do fígado e do cérebro, diminuição do conteúdo protéico e do DNA, bem como dos níveis de glicogênio hepático (sem alterar os lipídeos destes tecidos), elevação de colesterol, ácidos graxos e de b-hidroxibutirato na circulação; hipoglicemia e hiponatremia. 43

\section{Alterações hormonais}

Estudo conduzido em coelhos 47 evidenciou um bloqueio na liberação de oxitocina pelo etanol, fato também observado em pesquisas com ratos. 48,49

Com relação a prolactina (PRL) os resultados são ainda conflitantes. Alguns autores notaram níveis plasmáticos aumentados com valores reduzidos do hormônio luteinizante (LH), após administração intragástrica de álcool em animais por três dias 50 ou cronicamente através de dieta líquida por cinco semanas. 51

Subrananian et al.52 por outro lado demonstraram que a administração de álcool na forma aguda, no mesmo modelo experimental, não altera os níveis basais de prolactina. No entanto inibiu por mais ou menos uma hora a liberação deste hormônio induzida pela sucção; posteriormente este autor sugeriu que a liberação da prolactina é restabelecida após a metabolização do álcool indicando que a inibição da liberação da PRL pela sucção está diretamente relacionada ao nível de alcoolemia.29,53 Não está claro o mecanismo de ação desta inibição, e vários estudos evidenciaram que o álcool atua na pituitária anterior. $25,28,54,55$

Quanto à insulina, não existem até o momento informações sobre o seu nível sangüíneo durante a ingestão de álcool exclusivo na lactação. No entanto, Vinas et al. ${ }^{56}$ estudando ratas alcoolizadas nos perío- 
dos de gestação e lactação observaram insulina e glicemia plasmáticas normais neste último.

\section{Alterações do metabolismo enzimático}

Valores em torno de $90 \%$ do etanol ingerido são metabolizados no fígado através das sucessivas oxidações, primeiro a acetaldeído e posteriormente a acetato, para entrar no ciclo dos ácidos tricarboxílicos. Embora sejam várias as enzimas que podem transformar o álcool em acetaldeído, quantitativamente a mais importante é a álcool desidrogenase (ADH), cujas isoenzimas podem diferenciar-se em três classes, sendo a classe I a mais efetiva nas concentrações habituais de etanol. Essas isoenzimas dependem de controle genético, hormônios, estado nutricional e de outros fatores ainda não bem estabelecidos. ${ }^{3}$

A enzima aldeído-desidrogenase, responsável pelo metabolismo hepático do acetaldeído, tem menor atividade em ratas lactantes comparadas com ratas virgens. Como conseqüência, ratas lactantes apresentam níveis de acetaldeído plasmático até 20 vezes maiores do que os valores de não lactantes. 57

Guerri e Sanchis 58 observam que os níveis de acetaldeído são $30 \%$ a $50 \%$ mais baixos no leite do que no plasma, e que ocorre aumento não só na captação desta substância pela glândula mamária ${ }^{59}$ como também na atividade de aldeído-desidrogenase. 5

Alguns estudos têm verificado que a concentração plasmática de etanol na prole de ratas alcoolizadas era bem menor do que aquela do leite e sangue das mães, sendo os níveis de acetaldeído plasmático praticamente indetectável.58,59 Tavaresdo-Carmo e Nascimento-Curi60 estudando neste mesmo modelo experimental, registram alterações no metabolismo materno, com decréscimo do conteúdo protéico e aumento da lipogênese na mama, provocando nos filhotes redução do cérebro, fígado e peso corporal, com decréscimo do conteúdo protéico no fígado e plasma. Evidencias dos mesmos autores mostraram preservação dos triglicerídeos nas mamas. 40 A atividade de enzima ATP-citrato-liase também sofre alterações hepáticas dependentes da concentração de álcool consumida.61

\section{Alterações nutricionais na lactação de animais alcoolizados}

Pesquisas realizadas com ratos no período de lactação sugerem que o consumo de etanol, até mesmo em baixas concentrações (5\%) afeta o estado nutricional da mãe e diminui o nível de retinol no leite materno. 39

O efeito da ingestão de álcool por quatro dias no período inicial (quinto ao oitavo dia) e médio (nono ao décimo segundo dia) de amamentação também provocou redução da lactação e baixo consumo de leite pelas crias. ${ }^{63}$ Alguns autores sugerem que esta resposta se deve à modificação imediata do aroma do leite. 32

Em animais cujas mães consumiram continuamente etanol na gestação e lactação, alterações na absorção intestinal de zinco no íleo distal são observadas no fim do período de aleitamento. 63 Nestas condições de alcoolismo, as mães são afetadas duplamente, apresentando redução ponderal e alteração na composição nutritiva do leite (elevação de fosfatidilserina); por outro lado não foram observadas mudanças no crescimento linear da ninhada. 53

Resultados diferentes foram encontrados por Detering et al.,22 estudando o efeito do etanol a 35\% administrado em uma dieta líquida consumida na gestação e lactação; ratos recém-nascidos mostraram retardo do crescimento físico (incluindo retardo do sistema nervoso central) em maior proporção do que as alterações causadas por privação nutricional.

Um importante problema nas pesquisas com álcool tem sido o efeito anorético da droga, resultando em depleção nutricional. Essa variável tem sido crucial nos estudos pré e pós-natais, porque a inadequada nutrição materna tem sido sempre confundida com os efeitos adversos do álcool.41

Diante do envolvimento do fator nutricional com o álcool, Lieber et al. ${ }^{14}$ desenvolveram um modelo para administração de álcool em animais, que possibilitou, pela primeira vez, demonstrar que o consumo do etanol produzia danos hepáticos, apesar do consumo de uma dieta nutricionalmente adequada em proteínas, vitaminas e minerais.

A partir deste modelo dietético pode-se observar que a quantidade de álcool no leite materno é inferior ao etanol consumido na bebida alcoólica da lactante. Por outro lado, Oyama et al. 45 observam que mesmo em baixas doses (5\%) esta exposição provoca alteração no metabolismo da glicose cerebral.

\section{Alterações comportamentais com etanol via leite materno}

Processos nutricionais e atividades comportamentais são afetados significantemente em ratas lactantes que consomem álcool, apesar da ingestão de dietas líquidas com teores protéicos recomendados para o modelo experimental durante a lactação.41,64 Essas 
alterações que também são observadas nos filhotes, são decorrentes de modificações na fisiologia do sistema nervoso central, observando-se prolongamento do tempo de sono,65,66 déficit em áreas motoras, na área de aprendizado de habilidades e na área da memória; 44 alteração na concentração de galactolipídeos no cérebro e na medula espinhal devido ao aumento no conteúdo de lipídeos na bainha de mielina, também tem sido documentadas. 67

\section{Crescimento e desenvolvimento}

Padrões normais de crescimento e desenvolvimento são alterados na prole de mães que consumiram álcool durante o aleitamento, 41,44 inclusive com declínio da relação peso do coração/peso corporal, sugerindo uma ação do etanol na supressão do crescimento dos tecidos cardíacos, por declínio na freqüência mitótica. 68,69

\section{Referências}

1. Mitchell MC, Herlong HF. Alcohol e nutrition: caloric value, bioenergetics, and relation ship to liver damage. Annu Rev Nutr 1986; 6: 457-74

2. Galduroz JC. 1o Levantamento domiciliar nacional sobre uso de drogas psicotrópicas. São Paulo: Universidade Federal de São Paulo, 2000. [no prelo].

3. Mitchell MC. Alcohol. In: OPS (Organización Panamericana de la Salud). Conocimientos actuales sobre nutrición. Washington, DC: OPS; 1991. p. 530-4.

4. Halsted, C.H. Alcohol: efectos clínicos y nutricionales. In OPS (Organización Panamericana de la Salud). Conocimientos actuales sobre nutrición. Washington, DC: OPS; 1997. p. 584-593.

5. Mcginnis JR, Foege WH. Actual causes of death in the United States. JAMA 1993; 270: 2207-12.

6. Halsted CH, Robles EA, Mezey E. Decreased jejunal uptare of labelled folic acid (3H-PGA) in alcoholic patients: roles of alcohol and nutrition. N Engl J Med 1971; 285 : 701-6.

7. Hammoumi S, Naassila M, Daoust M. Experimental findings in the study of the reduction of alcohol intake. Eur Neuropsychopharmacol 1997; 7 Suppl 3: 337-40.

8. Prentice AM. Alcohol and obesity. Int J Obes 1995; 19: 4450.

9. Souza GT. Rodrigues MC, Ciavaglia MC. Analysis of the level of knowledge in the population of the teratogenic. Rev Bras Enferm 1996; 49: 287-304.

10. Volpi E. Moderate and large doses of ethanol differentially affect hepatic protein metabolism in humans. J Nutr 1998; 128: 198-203.

11. Wilson WR, Phillips KS, Leslie SW. Ethanol and glutamate effects of intracellular magnesium. Alcohol 1997; 14 533-6
Efeitos sobre o desenvolvimento do cérebro são observados com ingesta alcoólica no pré-natal.70

\section{Considerações finais}

Os resultados obtidos nos estudos com seres humanos e em vários modelos animais nos levam a concluir que o álcool predispõe a um grande número de alterações hormonais, metabólicas e fisiopatológicas que repercutem gravemente no desenvolvimento e crescimento dos filhos.

Seguramente o reconhecimento do problema pelos profissionais de saúde que atuam junto às gestantes e lactantes poderá representar um avanço na redução da mortalidade e morbidade dos recémnascidos de gestantes e/ou lactantes que inadvertidamente, ou até seguindo orientações inadequadas, tenham consumido bebidas alcóolicas.
12. Schuckit MA. Álcool e alcoolismo. In: Harrison Medicina interna. 11. ed. Rio de Janeiro: Guanabara-Koogan; 1988. v. 2, p. 1948-53

13. Lieber CS, Spreitz N, Decarli LM. Role of dietary adipose, and endogenously synthezid faty liver. J Clin Invest 1966, 45: 51-62.

14. Lieber CS, Jones DP, Decarli LM. Effects of prolonged ethanol intake: production of faty liver despite adequate diets. J Clin Invest 1965; 44: 1009-21.

15. Streissguth AP, Landesman-Dwyer S, Martin JC, Smith DW. Teratogenic effects of alcohol in humans and laboratory animals. Science 1980; 209: 353-61.

16. Warner RH, Rosett HL. The effects of drinking on off spring an historical survey of the American and Britishliterature. J Stud Alcohol 1975; 36: 1395-420.

17. Gallo PV, Weinberg J. Organ growth and celular development in ethanol exposed rats. Alcohol 1986; 3: 261-7.

18. Mena MA, Zorzano A, Herrera E. Acute effects of ethano on braian, plasma and adrenal monoamine concentration in virgin and pregnant rats and their fetuses. Nev Rochem Int 1986; 9: 371-8.

19. Sanchis R, Sanchotelo M, Chirivella M, Guerri C. The role of maternal alcohol-damage on ethanol teratogenicity in the rat. Teratology 1987; 36: 199-208.

20. Jones KK, Smith DW, Ulleland CN, Streissguth AP. Pattern of malformation in off spring of chronic alcoholic mothers. Lancet 1973; 1: 1267-71.

21. Tavares-do-Carmo MGT, Nascimento CMO, Martin A, Herrera E. Ethanol intake during lactation impairs milk production in rats and affects growth and metabolism of suckling pups. Alcohol 1999; 18: 71-6.

22. Detering N, Reed WD, Ozand PT, Karahasan A. The effects 
of maternal ethanol consumption in the rat on the development of their offspring. J Nutr 1979; 109: 999-1009.

23. Menella JA. The transfer of alcohol to human milk. N Engl J Med 1991; 325: 981-5.

24. Menella JA. Effects of beer on brestfed infants. JAMA 1993; 269: 1637-38

25. Subramanian MG. Prolactin secretion in lactatino rats following chronic alcohol exposure-provacative tests with secretagogues. Life Sci 1995; 57: 533-9.

26. Subramanian MG. Effects of chronic alcohol administration on lactational performance in the rat. Alcohol 1995; 12: $137-43$.

27. Subramanian MG. Alcohol inhibits suckling-induced oxytocin release in the lactating rat. Alcohol 1999; 19: 51-5.

28. Subramanian MG, Abel EL. Alcohol in hibits suckling-induced prolactim-release and milk-yield. Alcohol 1988; 5: 95-8.

29. Subramanian MG. Inhibitory effect of alcohol on the established suckling-induced prolactin surge in lactating rats. Proc Soc Exp Biol Med 1991; 198: 579-83.

30. Liston J. Breastfeeding and the use of recreational drugs-alcohol, caffeine, nicotine and marijuana. Breastfeed Rev 1998; 6: 27-30

31. Gottsfeld A, Legrue SJ. Lactational alcohol exposure elicits long-term immune deficits and increased noradrenergic synaptic transmission in lymphoid organs. Life Sci 1990; 47: 457-65.

32. Menella JA, Beauchamp GK. Short-term effects of maternal alcohol consumption on lactational performance. Alcohol Clin Exp Res 1998; 22: 1389-92.

33. Seelig LL Jr, Steven WN, Stewart GL. Second generation effects of maternal ethanol consumption on immunity to trichinella spiralis infemale rats. Alcohol Alcohol 1994; 34: $520-8$.

34. Grossman E. Beer and breast feeding: the wisdow of old wines. JAMA 1988; 259: 1096

35. Na AR, Daniels LC, Seelig LL Jr. Preliminary study of how alcohol consumption during pregnancy affects immune components in breast milk and blood of postpartum women. Alcohol Alcohol 1997; 32: 581-9.

36. Zordano A, Herrera E. Decreased in vivo rate of ethanol metabolism in the suckling rat. Alcohol Clin Exp Res 1989; 13: 527-32.

37. Silva VA, Malheiros LR, Moraes-Santos AR, Barzano MA, McLean AE. Ethanol pharmacokinetics in lactating women. Braz J Med Biol 1993; 26: 1097-103.

38. Lawton ME. Alcohol in breast milk. Aust N Z J Obstet Gynaecol 1985; 25: 71-3.

39. Albuquerque KT, Ramalho RA, Soares AG, Tavares-doCarmo MG. Effects of ethanol intake on retinol concentration in the milk of lactating rats. Braz J Med Biol Res 1998; 31: 929-32.

40. Tavares-do-Carmo MG, Nascimento-Curi CMO. Effects of ethanol intake on lipid metabolism in the lactating rat. Alcohol 1996; 13: 443-8.

41. Sanchis R, Sancho-Tello M, Guerri C. The role of liquid diet formulation in the postnal ethanol exposure of rats via mother's milk. J Nutr 1989; 119: 82-8.

42. Goodlett CR Mercussen BL, West JR. A single day of alcohol exposure during the brain growth spurt induces brain weight restriction and Purkinje cell loss. Alcohol 1990; 7. $107-14$
43. Neves J, Faccin GL, Tavares-do-Carmo MG. Efeito da in gestão de álcool durante a lactação sobre a produção e composição do LM e sobre o crescimento da prole: estudo em ratas. R. Nutr PUCAMP 1995; 8: 47-64.

44. Vaglenova J, Petkov VV. Fetal alcohol effects in rats exposed pre-and postnatally to a low of ethanol. Alcohol Clin Exp Res 1998; 22: 697-703.

45. Oyama LM, Couto RC, Couto GEC, Damaso AR, Nascimento CMO. Ethanol intake during lactation-effects on dams' metabolism and pups' body weght gain. Alcohol 2000; 21: 195-200.

46. Abel CL. Alcohol ingestion in lactating rats: effects on mothers and offspring. Arch Int Pharmacod 1974; 210: 121-127.

47. Fuchs AR, Wagner G. The effect of ethyl alcohol on the release of oxytocin in rabbits. Acta Endocrinol 1963; 44: 593-605

48. Fuchs AR. Etanol and the inhibition of oxytocin release in lactating rats. Acta Endocrinol 1969; 62: 546-54

49. Lincoln DW. Milk efection during alcool anaesthesia in the rat. Nature 1973; 243: 227-9.

50. Dees WL, Kozlowski GP. Differential effects of ethanol on luteinizing hormone, follicle stimulating hormone and prolactin secretion in the female rat. Alcohol 1984; 1 : 429-33.

51. Sanchis R, Esquifino A, Guerri C. Chronic ethanol intake modifies estrous cy clicity and alters prolaction and $\mathrm{LH}$ levels. Pharmacol Biochem Behav 1985; 23: 224-231.

52. Subramanian MG, Chen XG, Bergeski BA. Pattern and duration of the inhibitory effect of alcool administered acutely on suckling-induced prolactin rats. Alcohol Clin Exp Res 1990; 14: 771-5.

53. Heil SH, Hungund BL, Heng HH, Jen KLC, Subramanian MG. Ethanol and lactation: efeccts on milk lipids and serum constituents Alcohol 1999; 18: 43-8.

54. Subramanian MG. Beta-endorphin-stimulated prolactin-release in lactanting rats following alcohol administrarion. Alcohol 1994; 11: 269-72.

55. Subramanian MG, Savoymoore RT. Alcohol effects on TRH-induced prolactin response in lactating rats - in vivo and in vitro studies. Alcohol 1993; 10: 11-5.

56. Viñas O, Villaró S, Remesar X, Herrera E. Effects of chronic ethanol ingestion on circulating metabolites and liver composition in the lactating rat. Gen Pharmacol 1986; 17: 197-202.

57. Gordon BHJ, Baraona E, Lieber CS. Blood acetaldehyde response to ethanol ingestion during the reproductive cycle of de female rat. Alcohol 1985; 2: 271-5.

58. Guerri C, Sanchis R. Alcohol and acetaldehyde in rat's milk following ethanol administration. Life Sci 1986; 38: 1543-56

59. Villaró S, Viñas O, Herrera E, Remesar X. Chronic and acute ethanol impair the "in vivo" glucose uptake by lactating rat mammary gland. Biosci Rep 1987; 7: 777-80.

60. Tavares-do-Carmo MG, Nascimento-Curi CMO. Effect of ethanol intake during lactation on the metabolism on dams and on pup development. Braz J Med Biol Res 1990; 23: 1161-3

61. Oyama LM, Couto RC, Couto GE, Dâmaso AR, Oller do Nascimento CM. Ethanol intake during lactation. II. Effects on pups' liver and brain metabolism. Alcohol 2000; 21:201-6 
62. Subramanian MG. Evaluation of lactational parameters af ter alcohol administration for four days during early or midlactation in the rat. Alcohol Clin Exp Res 1997; 21 799-803.

63. Tavares E, Carreras O, Gómez Tubio A, Herce Pagliali C Murillo ML. Zinc intestinal absorption in newborn rats at 21 day postpartum: effects of maternal ethanol consumption. Life Sci 1988; 62: 787-97.

64. Daniel MA, Evans MA. Quantitative comparison of maternal ethanol and maternal tertiary butanol diet on postnatal development. J Pharmacol Exp Ther 1982; 222: 294 300 .

65. Klintsova AY, Cowell RM, Swain RA, Napper RM, Goodlett CR, Greenough WT. Therapeutic effects of complex motor training on motor performance deficits induced by neonatal binge-like alcohol exposure in rats. I. Behavioral results. Brain Res 1998; 1: 48-61.

Recebido em 7 de maio de 2001

Versão final reapresentada em 21 de julho de 2002

Aprovado em 1 de agosto de 2002
66. Tagashira E, Matsukawa K, Urano T, Suzuki T, Yanaura S. Functional changes of the central nervous system in rat offspring treated with ethanol during lactating period Nippon Yakurigaky Zasshi 1984; 84: 345-52.

67. Lalitha T, Kumar K, Ramakrishnan CV, Telang SD. Effect of maternal alcohol consumption on the lipid composition of CNS in the offspring. J Neurochem 1988; 50 1346-51.

68. Fuseler JW. Maternal ethanol consumption induces transient compensatory hyperplasia of developing cardiac tissue in the neonatal rat. Alcohol Alcohol 1993; 28: 657-66.

69. Heil SH, Subramanian MG. Chronic alcohol exposure and lactation - extended observations, Alcohol 2000; 2: 12732

70. Sanchis R, Guerri C, Renaupiqueras J, Grisolia S. Effect of prenatal and postnatal alcohol intake on brain-development. Int J Dev Neurosci 1985; 3: 419-22. 